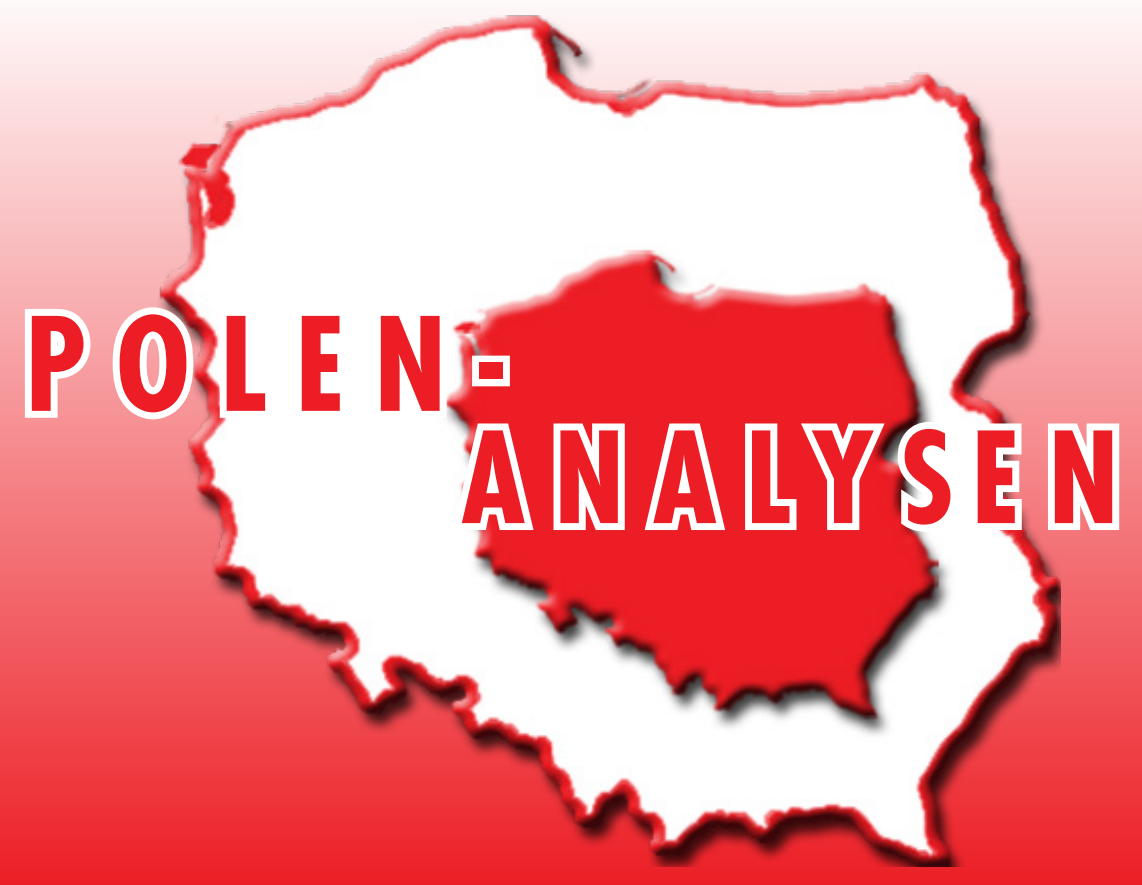

www.laender-analysen.de/polen

\title{
POLEN UND DER NATO-GIPFEL
}

\section{ANALYSE}

Säbelrasseln? Polen und der NATO-Gipfel

Reinhold Vetter, Warschau/Berlin

\section{CHRONIK}

21. Juni 2016 - 4. Juli 2016

Die nächste Nummer der Polen-Analysen erscheint nach der Sommerpause am Dienstag, den 6. September 2016.

Die Redaktion wünscht ihren Leserinnen und Lesern eine erholsame Sommerzeit.

Herausgegeben mit finanzieller Unterstützung

der Stiftung für deutsch-polnische Zusammenarbeit

FUNDACJA WSPÓŁPRACY

POLSKO-NIEMIECKIEJ

STIFTUNG

FÜR DEUTSCH-POLNISCHE

ZUSAMMENARBEIT

\section{DEUTSCHES POLEN INSTITUT}

- Forschungsstelle Osteuropa an der Universität Bremen
Deutsche Gesellschaft

DGO für Osteuropakunde e.V. 


\section{Säbelrasseln? Polen und der NATO-Gipfel}

Reinhold Vetter, Warschau/Berlin

\section{Zusammenfassung}

Der NATO-Gipfel am 8./9. Juli 2016 gehört zu den zentralen und immer wieder in die Öffentlichkeit getragenen Themen der Führung von Recht und Gerechtigkeit (Prawo i Sprawiedliwość- PiS) um Jarosław Kaczyński. NATOManöver wie »Anakonda 2016 « werden als "wichtiger Schritt in die richtige Richtung» gefeiert, damit Polen auch militärisch ein »gleichwertiges Mitglied» des Bündnisses werde. Doch die Politik der Nationalkonservativen geht weit über die Aspekte des rein Militärischen, der Landesverteidigung und der Abschreckung hinaus, die vom Bündnis weitgehend geteilt werden. Sie folgt einer militaristischen und emotionalisierenden Ideologie, die die Gesellschaft spaltet und die Kräfte an die Wand drängt, die für ein ziviles und tolerantes Miteinander eintreten.

Chon im Vorfeld der Präsidentenwahl und der ParOlamentswahlen am 10. Mai bzw. 25. Oktober 2015 hatten sich die Kandidaten der inzwischen regierenden PiS von Jarosław Kaczyński sowie ihr Bewerber für das Amt des Staatspräsidenten, Andrzej Duda, für eine deutliche Verstärkung der polnischen Armee sowie für die Einrichtung permanenter Militärbasen der NATO oder auch der USA in Polen eingesetzt. Dudas Berater Krzysztof Szczerski, seit August 2015 Staatssekretär in der Präsidialkanzlei, forderte die Bundesregierung in Berlin auf, ihren Widerstand gegen NATO-Basen in Polen aufzugeben und Polen in die Friedensverhandlungen zur Ukraine einzubinden.

In diesem Sinne wurde dann mit einer Ende März 2016 vom polnischen Parlament verabschiedeten und zwei Monate später vom neuen Präsidenten Duda unterzeichneten Gesetzesnovelle eine rechtliche Barriere für die dauerhafte Stationierung ausländischer Truppen in Polen auch in Friedenszeiten beseitigt. Diese Novelle hatten noch Abgeordnete der bis 2015 regierenden Bürgerplattform (Platforma Obywatelska - PO) angeregt. So stimmten dann im März dieses Jahres die Parlamentarier von $P i S$ und $P O$ fast einhellig für die Gesetzesänderung.

Seit dem Amtsantritt Dudas und dem Antritt der von $P i S$ geführten Regierung unter Ministerpräsidentin Beata Szydło ist der NATO-Gipfel am 8. und 9. Juli dieses Jahres ein permanentes Thema der polnischen Führung in ihrer politischen, propagandistischen und organisatorischen Arbeit. Sie feiert das bevorstehende Gipfeltreffen als Jahrhundertereignis und entsprechend soll es inszeniert werden. Während einer Pressekonferenz am 9. Juni nannte Verteidigungsminister Antoni Macierewicz das Treffen vollmundig den "größten, umfangreichsten und bedeutendsten Gipfel der NATO«. Auf der Homepage seines Ministeriums wurde der rumänische Verteidigungsminister Mihnea Motoc zitiert, der den Gipfel einen »Meilenstein« nannte. Wichtigster Veranstaltungsort wird das Nationalstadion in Warschau auf dem rechten Ufer der Weichsel sein, das neben der Sportarena auch große Konferenzräume beherbergt. Zur Inszenierung des 8./9. Juli wird des Weiteren eine Massenveranstaltung organisiert, deren "Austragungsort« jedoch noch nicht bekannt gegeben wurde.

Seit Mai sind die Schulen in ganz Polen angehalten, mehrere Unterrichtsstunden pro Woche dem euroatlantischen Bündnis und speziell dem Gipfel im Juli zu widmen. Die ganze Aktion wird von den Ministerien für Bildung, Verteidigung und Äußeres gesteuert. Besonders Gymnasiallehrer wurden aufgefordert, im Unterricht mit ihren Klassen ein Szenario durchzuspielen, bei dem die Einrichtung eines NATO-Stützpunktes in der jeweiligen Stadt oder Gemeinde simuliert wird. Außerdem sollen die Schüler mit den Einzelheiten der NATOEinsätze in verschiedenen Ländern vertraut gemacht werden, an denen polnische Soldaten beteiligt waren oder noch sind.

Dass die PiS-Regierung bei dieser militärpolitischen Instrumentalisierung der Schulen auch Unterstützung von Vertretern anderer politischer Richtungen erhält, zeigt das Beispiel des früheren Verteidigungsministers Janusz Onyszkiewicz, der sich bislang eher im MitteLinks-Spektrum engagiert hat. Die Tageszeitung Reczpospolita zitierte ihn am 26. Mai dieses Jahres mit der Feststellung, dass der "NATO-Unterricht" in den Schulen »eine gute Idee« sei. Onyszkiewicz sagte weiter: »Aber wichtig ist dabei, dass sich in den Schulen Lehrer finden, die den Schüler das Thema auf interessante Weise nahebringen. (...) Ich hoffe, dass der entsprechende Unterricht nicht so dargeboten wird, dass ihn die Schüler einfach über sich ergehen lassen und die Inhalte später schnell vergessen. (...) Dieser Unterricht stärkt nicht nur das Verteidigungsbewusstsein, sondern stärkt auch das Gefühl der transatlantischen Gemeinschaft«. Offensichtlich hat Onyszkiewicz bei seinen Äußerungen nicht bedacht, dass er damit auch die innenpolitische Instrumentalisierung des NATO-Gipfels durch Kaczyńskis PiS und die Regierung unterstützt, die den Wählern suggerieren will, dass sie die einzige politische Kraft in Polen ist, die das Land besonders im Zusammenwirken mit der NATO vor jedweder Aggression aus dem Aus- 
land schützen kann - ein Hohn auf verdiente polnische Politiker wie die verstorbenen Bronisław Geremek und Tadeusz Mazowiecki, die sich schon früh für den NATO-Betritt Polens eingesetzt hatten, der dann im Jahr 1999 erfolgte.

\section{Rückkehr Russlands zum Kalten Krieg?}

Wie sieht nun die Bedrohungsanalyse der regierenden Nationalkonservativen aus und welche Konsequenzen müssen ihrer Meinung nach daraus gezogen werden? Der PiS-Vorsitzende Jarosław Kaczyński gab die Richtung vor, als er am 7. April dieses Jahres in einem Interview mit der nationalkonservativen Zeitung Gazeta Polska Codzienna erklärte, dass die auf dem Gipfel zu erwartenden Entscheidungen zur Stärkung der Ostflanke der NATO dazu führen würden, dass Polen nicht nur politisch, sondern auch militärisch ein vollwertiges Mitglied des Bündnisses werde. Schon in einer außenpolitischen Grundsatzerklärung am 29. Januar 2016 hatte Außenminister Witold Waszczykowski betont, dass es in Bezug auf die Verteidigungsbereitschaft immer noch Disproportionen zwischen »alten " und »neuen « NATOMitgliedern gebe und sein Land deshalb gleiche Sicherheitsstandards aller Mitglieder des Bündnisses anstrebe. Bei einem Staatsbesuch in Dänemark im Juni dieses Jahres meinte Präsident Andrzej Duda, Russland sei ein Nachbar mit großem militärischem Potential. Moskau achte die Schwachen nicht, und deshalb müsse die NATO Geschlossenheit, Solidarität und Stärke zeigen. Bereits während der Münchener Sicherheitskonferenz im Februar 2016 hatte Duda festgestellt, Russland wolle eine Rückkehr des Kalten Krieges, um die nach 1989 entstandene internationale Ordnung in Frage zu stellen. Der konservative Publizist Andrzej Talaga forderte in der Rzeczpospolita, dass nicht nur US-Truppen, sondern auch deutsche und französische Einheiten nach Polen entsandt werden sollten. Der Moskauer Korrespondent der Tageszeitung Gazeta Wyborcza, Wacław Radziwinowicz, äußerte sogar die Vermutung, dass man in Moskau glaube, die NATO werde nicht entschieden reagieren, sollte Russland ins Baltikum vorrückten. Sprecher der PiS erhoben mehrfach die Forderung, die NATO-Russland-Akte müsse aufgekündigt werden, damit NATO-Einheiten leichter in Ländern wie Polen stationiert werden könnten.

Bei fast allen derartigen Stellungnahmen und Meinungsäußerungen fällt auf, dass sie sich fast ausschließlich auf die direkten militärischen Aspekte einer möglichen Bedrohung Ostmitteleuropas durch Russland beziehen, aber das gesamte Krisenszenario, das die Einheit Europas und speziell der EU bedroht, nicht ins Visier nehmen. Eine Ausnahme bildete ein umfangreicher Text von Außenminister Witold Waszczykowski am 4. April 2016 in der Frankfurter Allgemeinen Zeitung. Dort sprach er nicht nur von einer "aggressiven russischen Politik, die darauf abzielt, Einflusssphären zu erhalten, die europäische Sicherheitsarchitektur zu verändern und den Wandel in Staaten zu hemmen, die die Zusammenarbeit mit der EU intensivieren«. Vielmehr erwähnte er auch die Auswirkungen der Instabilität im Nahen Osten und Nordafrika sowie die erhöhte Terrorgefahr seitens islamistischer Gruppen sowie mögliche weitere Wellen illegaler Migration. Leider wurde dieser Text nicht auch in Polen veröffentlicht. Immerhin sprach Präsident Andrzej Duda im April im National Press Club in Washington davon, dass der bevorstehende NATO-Gipfel "universalen Charakter" haben und auf "alle Herausforderungen an das Bündnis" reagieren müsse.

Hinzu kommt, dass selbst Waszczykowski die Gesamtheit der "hybriden« Kriegsführung Moskaus in ganz Europa, wozu u. a. das aggressive Auftreten russischer Medien wie Russia Today und die Finanzierung von Rechtspopulisten wie dem Front National in Frankreich durch den Kreml gehören, nicht in den Blick nimmt geschweige denn die führenden Politiker der PiS, die sich einer solchen umfassenden Analyse vollständig verweigern bzw. intellektuell dazu nicht in der Lage sind. Schon in ihrer Regierungserklärung am 18. November 2015 hatte Ministerpräsidentin Beata Szydło die schweren internationalen Krisen und deren Implikationen für Europa nur ganz am Rande oder gar nicht erwähnt.

Der renommierte politische Analytiker Aleksander Smolar, seit langem Direktor der Stefan Batory Stiftung (Fundacja im. Stefana Batorego) in Warschau, betonte in einem Interview mit Newsweek am 29. Mai dieses Jahres, dass gerade Jarosław Kaczyński ein krasses Beispiel dafür sei, wie sehr das außenpolitische Denken der PiSFunktionäre von Ratlosigkeit und einem anachronistischen Blick auf die internationalen Beziehungen insbesondere in Europa geprägt sei. Über die polnische Geschichte, so Smolar, wisse Kaczyński mit Sicherheit viel mehr als über die heutige Situation. Seine Vorstellung von Außenpolitik beziehe sich auf geopolitische Traditionen des 19. Jahrhunderts und der Zeit zwischen den beiden Weltkriegen - das heißt unaufhörliche Kämpfe zwischen den Nationalstaaten, eine Welt, in der Chaos herrscht, in der die Großen die Kleinen dominieren wollen und die Kleinen Koalitionen gegen die Großen bilden. Smolar erläuterte: „Ein großer Teil des Widerwillens, den Kaczyński gegenüber Deutschland hegt, resultiert aus dem Imperativ, Widerstand gegen das stärkste Land in Europa zu organisieren, das Dominanz über andere Staaten anstrebt. Ihre (die der PiS-Funktionäre-R.V.) gesamte Rhetorik des ssich von den Knien Erhebensı legt den Verdacht nahe, dass die- 
jenigen, die Polen heute regieren, ein emotionales Problem mit ihrem eigenen Ehrgefühl haben."

Wiederholt hat Kaczyński betont, dass der Westen nach wie vor gegenüber Polen bestimmte Verpflichtungen habe, die aus dem Zweiten Weltkrieg und den Beschlüssen der Konferenz in Jalta im Februar 1945 resultieren, in der sich Franklin D. Roosevelt, Winston Churchill und Josef Stalin über die künftige Machtverteilung in Europa verständigt hatten. Kaczyński vergisst dabei u. a., dass führende polnische Politiker wie der verstorbene Außenminister Bronisław Geremek den Beitritt Polens zur NATO im Jahr 1999 als endgültige Überwindung des Jalta-Systems gewürdigt hatten. Antoni Macierewicz, heute Verteidigungsminister, verstieg sich sogar zu der Bemerkung, dass Länder, die gerade einmal seit 200 Jahren über Erfahrungen mit der Demokratie verfügten, gegenüber Polen nicht als Lehrmeister auftreten sollten.

Der Schlüssel zum Verständnis der Außenpolitik von $P i S$, so Smolar, liege in ihrer Innenpolitik. Wenn international das Ansehen Polens gesunken sei, dann resultiere das nicht in erster Linie aus den außenpolitischen Äußerungen von Kaczyński und Macierewicz, sondern vor allem aus der Tatsache, dass PiS die demokratischen Fundamente Polens schwäche, aus ihrem rabiaten, den Rechtsstaat, die staatliche Gewaltenteilung und die Meinungsfreiheit missachtenden Umgang mit dem Verfassungsgericht, der Justiz insgesamt und den Medien.

Die weitgehend stabilen Umfragewerte für PiS sind möglicherweise auch ein Hinweis darauf, dass ihre militärische Bedrohungsanalyse von einem beträchtlichen Teil der polnischen Gesellschaft geteilt wird. Schon ihr Sieg bei den Parlamentswahlen im Jahr 2015 resultierte ja nicht zuletzt aus den Ängsten vieler Bürger, internationale Einflüsse militärischer, ökonomischer und kultureller Art könnten sie existentiell bedrohen.

\section{PiS fehlt Verständnis für »hybride Kriegsführung"}

Es lohnt sich zu vergleichen, wie die NATO die Politik der russischen Führung um Wladimir Putin analysiert, wobei die Einschätzung innerhalb des Bündnisses nicht ganz einheitlich ist. Jedoch lassen sich gewisse Gemeinsamkeiten feststellen. So herrscht die Auffassung vor, dass die Annexion der Krim völkerrechtswidrig ist und die gezielte Herbeiführung eines gewaltsamen Konflikts in der östlichen Ukraine durch die Installierung von Separatisten kriegerische Gefahren auch wieder nach Europa gebracht haben.

Mit Sorge werden auch die "weichen«, nicht-militärischen Methoden russischer Einflussnahme im postsowjetischen Raum und in der Europäischen Union betrachtet - sei es durch russische Auslandsmedien wie
Sputnik oder RT (ehemals Russia Today), die gezielte Ausweitung von Informationsnetzwerken in der Wirtschaft oder die Finanzierung links- und rechtspopulistischer europakritischer Parteien und Organisationen in der EU. Gesprochen wird von einem russischen »Informationskrieg« zur Beeinflussung öffentlicher Debatten in den Mitgliedsländern der Gemeinschaft. Bei ihrem Treffen Mitte Juni 2016 erklärten die NATO-Verteidigungsminister zudem, Angriffe über Datennetze würden künftig wie solche durch Land-, See- und Luftstreitkräfte behandelt. Das könne bedeuten, dass solche "Cyber-Angriffe» den Bündnisfall nach Artikel 5 des NATO-Vertrages auslösen könnten.

Die Benennung der internationalen Krisensymptome wäre nicht vollständig ohne den Verweis auf die Tatsache, dass Russland und auch die USA ein Vierteljahrhundert nach dem Ende des Kalten Krieges an einer Modernisierung ihrer Atomwaffen arbeiten. Russland besitzt heute nach wie vor ein mit den USA vergleichbares Arsenal an Atomwaffen. Doch vergleicht man alle der NATO zur Verfügung stehenden militärischen Potentiale mit den entsprechenden Fähigkeiten Russlands, dann erkennt man eine klare Überlegenheit der NATO, die bei Konflikten mit NATO-Staaten in der unmittelbaren russischen Nachbarschaft eine Rolle spielen könnte.

Bekannt ist, dass die russische Führung nach eigenen Aussagen ihre innenpolitischen Repressionen gegen die ersten Ansätze einer Zivilgesellschaft sowie gegen unabhängige bzw. oppositionelle Gruppierungen, die Kontakt zum Westen haben und von dort gefördert werden, als Antwort auf die Instrumente und Methoden ansieht, die »der Westen" selbst in Russland und in den postsowjetischen Staaten zum Zweck der Demokratieförderung als legitim ansieht. Im Kreml herrscht tatsächlich die Auffassung vor, dass insbesondere die USA mit sozialen Netzwerken, Jugendorganisationen und NGOs die innere Entwicklung in Russland und den postsowjetischen Staaten beeinflussen und destabilisieren wollen.

In der Brüsseler NATO-Führung dominiert die Einschätzung, dass der enge Kreis um Putin aus Personen des Sicherheitsapparats besteht, die mehrheitlich noch im sowjetischen Geheimdienst ausgebildet wurden und ihr Verständnis von Sicherheit und den eigenen Machterhalt über die wirtschaftlichen Interessen des Landes stellen. Das Denken dieser Leute sei bis heute von den Stereotypen des Kalten Krieges geprägt und tief in der früheren sowjetischen Propaganda verwurzelt, die Antiamerikanismus mit Einkreisungsängsten verband. Deutlich sei das russische Streben, eine Großmachtstellung einzunehmen. Es beinhaltet das Streben nach einer internationalen Ordnung, in der die USA und Russland einen prominenten Platz haben und in 
der Russland sich nicht gegen (als solche wahrgenommene) von außen geförderte Unruhen und Revolutionen wehren muss. Der frühere außenpolitische Sprecher der SPD-Bundestagsfraktion, Karsten D. Voigt, schrieb in einem Beitrag für die Russland-Analysen Nr. 295 (2015): "Die Gründe für die außenpolitische Neuorientierung Russlands liegen in der Innenpolitik: Präsident Putin betrachtet den Zerfall der Sowjetunion und das Ende des Sowjet-Kommunismus nicht als historische Chance für den Aufbau eines modernen und demokratischen Russland, sondern als sgrößte geostrategische Katastrophe des zwanzigsten Jahrhunderts،. Putins Russland will international nicht als das Land anerkannt werden, das es heute ist, sondern als das, was es einmal war: als Imperium. Das Streben nach Erhalt und Wiedergewinnung von Einflusszonen wird von den meisten Nachbarn als russischer Revisionismus wahrgenommen.«Schon in der russischen Militärdoktrin von 2010 wurde die NATO als eine der Hauptbedrohungen für Russland charakterisiert. Mitte Juni 2016 ließ Präsident Putin aufhorchen, als er bei einem Wirtschaftsforum in Sankt Petersburg erklärte, dass die USA vermutlich die letzte verbliebene Supermacht seien und man das akzeptiere. Ist das ein Sinneswandel oder pure Taktik?

Es bleibt die Frage, ob Polen von Russland militärisch bedroht ist. Wohl eher nicht. Andererseits tangiert die Gesamtheit der Aktionen der "hybriden Kriegsführung « Russlands auch Polen. Polens Außenminister Witold Waszczykowski beantwortete die Frage auf seine Weise, indem er erklärte, dass Russland eine größere Gefahr für Europa darstelle als die Terrorgruppen des "Islamischen Staates".

\section{Balanceakt oder Säbelrasseln?}

Ausgehend von ihrer in den letzten Monaten vorgenommenen Einschätzung der Lage ist die NATO Ländern wie Polen inzwischen ein gutes Stück entgegengekommen. So beschlossen die NATO-Verteidigungsminister Mitte Juni 2016 die rotierende Stationierung von je einem Bataillon in Polen, Litauen, Lettland und Estland - ein Beschluss, der allerdings noch beim NATOGipfel im Juli bestätigt werden muss. Deutschlands Verteidigungsministerin Ursula von der Leyen gelang die Wortschöpfung, dass es sich dabei um eine »Vorne-Präsenz" handeln werde. Danach geht es um einen Balanceakt zwischen Abschreckung und nicht zu martialischem Auftreten. Die etwa 1.000 NATO-Soldaten je Land sollen etwa alle neun Monate wechseln. Auf diese Weise soll die NATO-Russland-Akte von 1997 eingehalten werden, mit der die NATO zugesagt hatte, dass sie »im gegenwärtigen und vorhersehbaren Sicherheitsumfeld keine zusätzlichen substantiellen Kampftruppen dauerhaft stationiert (in den östlichen NATO-Staaten -
R.V.)«. Jedes Bataillon soll von einer "Rahmennation" geführt werden, wobei die USA diese Rolle in Polen und Deutschland in Litauen übernehmen dürften.

Des Weiteren erklärte Philip Breedlove, Kommandeur des Europäischen Kommandos der US-Streitkräfte, man wolle von Februar 2017 an eine amerikanische Panzerbrigade in den Osten Europas verlegen. Die 4.200 Soldaten sollen rotierend für jeweils neun Monate eingesetzt werden und - etwa in den baltischen Staaten - an Übungen teilnehmen. Mitte Mai wurde außerdem im nordpolnischen Redzikowo, östlich von Stolp (Słupsk), der Grundstein für einen Stützpunkt des elektronischen Warn- und Feuerleitsystems »Aegis« der NATO, eine Art europäischamerikanische Raketenabwehr, gelegt. Ab 2018 sollen dort 300 amerikanische Soldaten stationiert werden. Offiziell heißt es, dieses System richte sich nicht gegen Russland, sondern diene der Abwehr von Raketen aus "Schurkenstaaten«. Diese Bewertung wird jedoch von Russland nicht geteilt. Die verschiedenen Schritte der NATO und der USA veranlassten den polnischen Außenminister Witold Waszczykowski zu der Bemerkung, man sei auf gutem Wege, »den ungleichen Sicherheitsstatus alter und neuer NATO-Mitglieder auszugleichen".

Ein Paukenschlag besonderer Art war natürlich das große Manöver »Anakonda 2016« im Mai dieses Jahres in Polen, das hauptsächlich von amerikanischen und polnischen Einheiten bestritten wurde, an dem aber auch Soldaten aus 15 weiteren NATO-Staaten sowie aus NATO-Partnerstaaten wie Georgien und der Ukraine, zudem aus Schweden und Finnland teilnahmen. Insgesamt waren mehr als 30.000 Soldaten im Einsatz. Auch 2.900 Panzer und andere Militärfahrzeuge, mehr als 100 Flugzeuge und Hubschrauber sowie zwölf Schiffe wurden eingesetzt. Das Szenario des Manövers umfasste klassische militärische Elemente wie Luftlandeoperationen und schnelle Truppenverlegungen, aber auch die Abwehr von Cyber-Angriffen und anderer Bedrohungsmuster hybrider Kriegsführung. Auch die Bundeswehr war beteiligt; ein Panzerpionierbataillon aus Minden mit 400 Soldaten baute eine 400 Meter lange Pontonbrücke über die Weichsel.

Das Manöver rief allerdings unterschiedliche Reaktionen hervor. So kritisierte Bundesaußenminister FrankWalter Steinmeier »Anakonda 2016« und forderte stattdessen mehr Dialog und Kooperation mit Russland. „Was wir jetzt nicht tun sollten, ist durch lautes Säbelrasseln und Kriegsgeheul die Lage weiter anzuheizen", sagte er der Bild am Sonntag und rief damit äußerst kritische Stimmen bei östlichen Nachbarn wie Polen, aber auch in Deutschland besonders bei der CDU hervor, während er von den Grünen und der Linkspartei starke Unterstützung erhielt.

Allerdings hatte Bild am Sonntag nur einen Teil des Statements veröffentlicht, das der Außenminister dem 
Blatt hatte zukommen lassen. In dem nicht veröffentlichten Teil hieß es: "Mit der Krim-Annexion und den militärischen Aktivitäten in der Ost-Ukraine hat Russland bei unseren östlichen Nachbarn ein Gefühl der Bedrohung entstehen lassen. Deswegen ist es richtig gewesen, eine gemeinsame Reaktion der NATO zu finden. Die Allianz weicht ihrer Verantwortung nicht aus. Niemand kann den (...) Umfang der NATO-Maßnahmen als Bedrohung für Russland werten." Das Problem war, dass das Auswärtige Amt den gesamten Umfang des Statements erst einen Tag, nachdem die Bild am Sonntag Aufsehen erregt hatte, veröffentlichte. Das legt die Vermutung nahe, dass Steinmeier mit seiner Äußerung über "Säbelrasseln und Kriegsgeheul« durchaus Aufsehen erregen wollte. Wenn das der Fall ist, dann hat der Außenminister die Risse in der NATO deutlich gemacht und ausgerechnet jenen Auftrieb gegeben, die - ganz im Sinne der Moskauer Propaganda - nicht in Russland, sondern in der NATO den Aggressor sehen.

Allerdings gab es selbst im NATO-Hauptquartier Stimmen, die meinten, das Manöver sei "viel zu plump auf Russland gemünzt«. Zudem sei das Durchspielen des Bündnisfalles so kurz vor dem NATO-Gipfel in Polen "zu dick aufgetragen«. Das Verhältnis zu Russland, so hieß es, sei in der Tat schwierig, mit dem Manöver sei allerdings »der Ernstfall ohne Not durchexerziert« worden. Der Russlandbeauftragte der Bundesregierung, Gernot Erler (SPD), wiederum sagte, die Abfolge der Übungen in osteuropäischen NATO-Ländern sei »unerhört dicht" geworden. Das reflektiere die Verunsicherung der östlichen NATO-Partner. Erler erinnerte auch daran, dass Russland im vergangenen Jahr Manöver mit insgesamt 95.000 Soldaten abgehalten hat.

Natürlich ließen Reaktionen aus Russland nicht lange auf sich warten. So sagte der russische NATOBotschafter: "Wir ergreifen regelmäßig militärische Maßnahmen, um diese verstärkte Präsenz auszugleichen, die durch nichts gerechtfertigt ist." Ein Sprecher von Präsident Putin betonte, das Manöver trage "nicht zu einer Atmosphäre des Vertrauens und der Sicherheit bei«, es gebe noch immer »ein gegenseitiges Vertrauensdefizit«.

Auch in Russlands Staatsmedien war Steinmeier wie zu erwarten - ein vielzitierter Mann, natürlich nur mit den Äußerungen, die Bild am Sonntag veröffentlicht hatte. Der Vorsitzende des Verteidigungsausschusses der Duma, Wladimir Komojedow, kündigte an, man werde weitere Zehntausende Soldaten im Süden und Westen Russlands stationieren. Außerdem wurden die seit 2013 praktizierten "unerwarteten Überprüfungen der Mobilisierungs- und Gefechtsbereitschaft « des russischen Militär nach dem NATO-Manöver in Polen noch einmal verstärkt.
Allerdings gibt es auch Hinweise darauf, dass die NATO und Moskau weiter im Gespräch bleiben wollen. So tagte erstmals seit 2014 wieder der NATO-Russland-Rat.

\section{Die Agenda des Gipfels}

Am 8. und 9. Juli wird man sich in Warschau vor allem bemühen, die Risse in der NATO zu kitten und angesichts der unterschiedlichen regionalen Bedrohungen einen Interessenausgleich zwischen den Bündnismitgliedern im Osten und Süden zu schaffen. Voraussichtlich bestimmen fünf Themenfeld die Agenda des Treffens: die Situation in Ostmittel- und Osteuropa, die Gefahren aus Nahost, die Zukunft der Partnerschaftspolitik, die Frage künftiger Erweiterungen des Bündnisses und die nukleare Rüstung.

Dabei werden die einzelnen NATO-Mitglieder unterschiedliche Prioritäten setzen:

- Die Mitgliedsstaaten im Osten Europas werden vor allem darauf dringen, die Abschreckungs- und Verteidigungsfähigkeit der NATO gegenüber Russland weiter auszubauen.

- Die südlichen Länder wollen verhindern, dass sich die NATO zu sehr auf den Osten konzentriert, und verlangen, dass die "Schnelle Eingreiftruppe» (Very High Readiness Joint Task Force) auch der Verteidigung Südeuropas dient.

- Länder wie Deutschland, Frankreich und Großbritannien wiederum werden versuchen, einen Ausgleich zu finden, um diese Spannungen zwischen "Ost" und »Süd« abzubauen.

- Die USA schließlich werden darauf dringen, dass die NATO insgesamt noch größere militärische Anstrengungen unternimmt.

- Vermutlich wird man auch über eine engere Kooperation zwischen der NATO und der EU verhandeln; eine entsprechende Erklärung ist in Vorbereitung.

\section{Stärkung der Armee, »Territorialverteidigung «", patriotische Mobilisierung}

Parallel zur stärkeren Verknüpfung der polnischen Armee mit der NATO betreibt die nationalkonservative Regierung in Warschau einen Ausbau der Streitkräfte. Verteidigungsminister Antoni Macierewicz kündigte an, dass allein das Heer von derzeit 100.000 auf 150.000 Mann aufgestockt werde. Des Weiteren plant der Minister eine Modernisierung der Armee; hier steht der Kauf von Mehrzweckhubschraubern auf Platz eins der Prioritätenliste. Dabei wird das Kabinett vermutlich einen noch von der Vorgängerregierung geschlossenen Vertrag über den Erwerb von 50 Hubschraubern des Typs "Caracal« des deutsch-französisch-spanischen Konzerns Airbus Helicopters zurückziehen. Ein neuer 
Vertrag könnte mit dem US-Mitbewerber Sikorsky ausgehandelt werden, der seine »Black Hawk« Hubschrauber im Gegensatz zu Airbus zum Teil auch in Polen produziert. Wiederholt haben Regierungsvertreter erklärt, dass das Kabinett derlei Aufträge lieber an Firmen vergibt, die auch in Polen Fabriken unterhalten. Geplant ist außerdem die Anschaffung von Flugabwehrraketen sowie eine Modernisierung von etwa 130 Panzern des Typs »Leopard«, wobei naturgemäß auch der deutsche Hersteller beteiligt wäre.

Seit dem Amtsantritt von Macierewicz dreht sich auch das Personalkarussell im Militär. So entband der Minister wenige Tage nach seinem Amtsantritt den Chef des Generalstabes und sieben weitere Generäle ihrer Posten, denen intern mangelnde Pflichterfüllung oder gar Mutlosigkeit vorgeworfen wurden. Macierewicz setzte im Sejm sogar eine Novellierung des Soldatengesetzes durch, die ihm die sprunghafte Beförderung von Militärs über mehrere Dienstgrade hinweg ermöglicht.

Das Lieblingsprojekt des Ministers ist die "Territorialverteidigung «, in die etwa 35.000 Mann aus Schützenverbänden, „Bürgerwehren« und anderen paramilitärischen Verbänden eingebunden und zur Unterstützung der Armee eingesetzt werden sollen. Schützenverbände und "Bürgerwehren" haben in Polen Tradition. Allein der Schützenverband Strzelec OSW JS 4046 aus Westpolen, dessen Existenz bis in das Jahr 1910 zurückreicht, umfasst mehr als 10.000 Mitglieder. In ganz Polen existieren inzwischen mindestens 130 »Bürgerwehren«. Einige dieser paramilitärischen Gruppen durften unlängst sogar an dem Militärmanöver »Anakonda 2016 « teilnehmen. Ihre Mitglieder, von denen viele mit der Regierungspartei Recht und Gerechtigkeit sympathisieren, sehen »ihre Stunde gekommen«. Intensiv pflegen sie das Andenken an die polnische Heimatarmee (Armia Krajowa), die während des Zweiten Weltkriegs gegen die deutschen Besatzer kämpfte und sich vor und nach 1945 auch der Roten Armee und dem kommunistischen Nachkriegssystem widersetzte.

Die Einbindung der Mitglieder von Schützenverbänden und "Bürgerwehren" in die "Territorialverteidigung« soll von September dieses Jahres in großem Stil erfolgen. Auf den ersten Blick erscheinen sie wie Freizeitsoldaten, die an den Wochenenden trainieren und mehrmals im Jahr auf Truppenübungsplätzen geschult werden. Experten sehen den militärischen Nutzen dieser paramilitärischen Einheiten eher kritisch. Doch nach dem Willen der Regierung und besonders von Minister Macierewicz sollen sie auch in Friedenszeiten "präventive Milizaufgaben « wahrnehmen und gegen »nichtmilitärische Bedrohungen« vorgehen. Man denkt dabei an »Masseneinwanderung aus den arabischen Ländern und Nordafrika», die zum »Anstieg organisierter Kri- minalität und zur Destabilisierung der staatlichen Verwaltung« führen könnte.

Fatale Erinnerungen an die in der Volksrepublik Polen bestehende Bürgermiliz (Milicja Obywatelska), wie damals die Polizei genannt wurde, und die Freiwillige Polizeireserve (Ochotnicza Rezerwa Milicji Obywatelskiej - ORMO), eine paramilitärische Truppe, die oft auch gegen oppositionelle Demonstranten und streikende Arbeiter eingesetzt wurde, werden wach. Die liberale und linke polnische Opposition hält die Begründung, mit der die Regierung die Einbindung der paramilitärischen Gruppen in die »Territorialverteidigung « rechtfertigt, für einen Vorwand. In Wirklichkeit, so heißt es, wolle Recht und Gerechtigkeit diese als private Schutztruppe für sich rekrutieren.

Besonders gefährlich ist die Tatsache, dass sich gerade auch rechtsradikale Gruppierungen wie das Nationalradikale Lager (Obóz Narodowo-Radykalny - ONR) mit großem Eifer in der "Territorialverteidigung" engagieren wollen und dies Minister Macierewicz nicht zu stören scheint. Die mehrere Tausend Mitglieder des ONR fallen insbesondere dadurch auf, dass sie martialische Aufmärsche organisieren, antisemitische Parolen verbreiten und ausländische Studenten bedrohen. Sie berufen sich bewusst auf das bereits in den späten 1930er Jahren existierende $O N R$, dessen Mitglieder sozialistische Studenten verprügelten, antisemitische Hetze betrieben, einen Numerus Clausus und besondere Sitzbänke für jüdische Studenten forderten und auch politische Morde verübten.

Insgesamt ist die Aufstellung der "Territorialverteidigung « Teil des Bemühens der Nationalkonservativen, die polnische Gesellschaft in ihrem politisch-ideologischen Sinne zu mobilisieren und zu militarisieren, wie auch am Beispiel des "NATO-Unterrichts» in den Schulen deutlich wird. Der für die Organisierung der »Territorialverteidigung« verantwortliche Grzegorz Kwaśniak erklärte in einer Rede: "Ihr Ziel ist die Stärkung der patriotischen und christlichen Fundamente unseres Militärs und unseres Verteidigungssystems, so dass Patriotismus und Glaube die beste Garantie für unsere Sicherheit sind."

\section{Fazit}

Es ist gut, dass der "polnische Blick" auf Russland, also eine Stimme aus dem östlichen Teil des integrierten Europas, in die europäische Sicherheitsdebatte getragen wird, auch wenn die Bedrohungsanalyse der Nationalkonservativen um Jarosław Kaczyński überzogen sein mag. Auch West- und Südeuropäer bringen ihren Standpunkt ein.

Für alle Europäer ist der Umgang mit einem Russland, von dem militärische Bedrohungen und andere Gefahren ausgehen, nicht einfach. Abschreckung, mili- 
tärische Übungen und Wachsamkeit gegenüber allen anderen Formen der "hybriden Kriegsführung" sind legitim. Aber ebenso muss das ganze Europa sowohl mit den Machthabern in Moskau als auch mit regionalen Repräsentanten und der Zivilgesellschaft Russlands im Gespräch bleiben. Gerade Polen könnte da viel aktiver sein. Solange die Machtverhältnisse in Russland so sind, wie sie sind, wird Russland immer ein Bedrohungsfaktor und eine Quelle von Instabilität sein, solange es sein Verständnis der Rolle eines eigenständigen und einfluss- reichen Akteurs in der internationalen Politik nicht ausreichend berücksichtigt sieht.

Das Fatale an der Politik der Nationalkonservativen in Polen in Bezug auf NATO und Russland besteht darin, dass sie weit über alle Aspekte des Militärischen, der Landesverteidigung und der Abschreckung hinausgeht. Sie folgt einer militaristischen Ideologie, die Polarisierung und Spaltung in die Gesellschaft trägt und alle Kräfte, die für ein friedliches und tolerantes Miteinander eintreten, an die Wand drängt.

\section{Über den Autor}

Reinhold Vetter ist freier Wissenschaftler und Publizist mit den Arbeits- und Forschungsschwerpunkten Zeitgeschichte und Politik Ostmittteleuropas. Er lebt in Warschau und Berlin. Seine jüngste Buchveröffentlichung »Bronisław Geremek. Der Stratege der polnischen Revolution« erschien 2014 in Berlin.

\section{CHRONIK}

\section{Juni 2016 - 4. Juli 2016}

\begin{tabular}{|c|c|}
\hline 21.06 .2016 & $\begin{array}{l}\text { Bei der Eröffnung der Konferenz des German Marshall Fund in Warschau »NATO’s Open Door Policy in War- } \\
\text { saw and Beyond« sagt der stellvertretende Außenminister Marek Ziółkowski Georgien und Mazedonien die } \\
\text { feste Unterstützung Polens für die Aufnahme in die NATO zu. }\end{array}$ \\
\hline 22.06.2106 & $\begin{array}{l}\text { In Berlin finden die XIV. Deutsch-Polnischen Regierungskonsultationen statt. Sie stehen im Zeichen des 25. Jah- } \\
\text { restags der Unterzeichnung des deutsch-polnischen Nachbarschaftsvertrags. Thematisiert werden die internatio- } \\
\text { nale Flüchtlingskrise und Lösungen, die mögliche Entscheidung Großbritanniens für einen EU-Austritt, Fra- } \\
\text { gen der Energiesicherheit, die bilaterale Zusammenarbeit in den Bereichen Wirtschaft, Infrastruktur, Jugend- } \\
\text { austausch und der Polnischunterricht in Deutschland. Auf einer Pressekonferenz, gefragt nach dem NATO- } \\
\text { Manöver "Anakonda 2016« in Polen vor zwei Wochen und dem Kommentar von Außenminister Frank-Walter } \\
\text { Steinmeier dazu, der vor "Säbelrasseln, Kriegsgeheul und symbolischen Paraden« warnte, dankt Ministerprä- } \\
\text { sidentin Beata Szydło dafür, dass Deutschland bei der Stärkung der NATO-Ostflanke Unterstützung leistet, } \\
\text { zumal jenseits der östlichen Grenze Krieg zwischen Russland und der Ukraine herrscht. Um über die Aufhe- } \\
\text { bung der EU-Sanktionen gegenüber Russland sprechen zu können, müsse zuerst das Minsker Abkommen zwi- } \\
\text { schen den Konfliktparteien umgesetzt werden. }\end{array}$ \\
\hline 23.06.2014 & $\begin{array}{l}\text { Ministerpräsidentin Beata Szydło empfängt in Warschau die Vizepräsidentin der Europäischen Kommission, } \\
\text { Kristalina Gieorgieva, Kommissarin für Haushalt und Personal. Thematisiert werden Fragen der Finanzen der } \\
\text { EU ab dem Jahr } 2020 \text { sowie die Finanzierung der Kohäsionspolitik und Landwirtschaftspolitik. Des Weite- } \\
\text { ren trifft sich die EU-Kommissarin mit Finanzminister Paweł Szałamach und dem stellvertretenden Außen- } \\
\text { minister Konrad Szymański. }\end{array}$ \\
\hline 24.06 .2016 & $\begin{array}{l}\text { Der Senat stimmt für eine weitere Gesetzesnovelle im Bereich der öffentlichen Medien. Demnach wird ein Rat } \\
\text { für die Nationalen Medien (Rada Mediów Narodowych) gebildet, der die Vorstände und Aufsichtsräte des Pol- } \\
\text { nischen Fernsehen, des Polnischen Radio und der Polnischen Presseagentur (TVP, PR, PAP) beruft. Dem Rat } \\
\text { gehören drei vom Sejm und zwei vom Präsidenten berufene Mitglieder an. Letztere werden von den jeweils zwei } \\
\text { größten Oppositionsparteien vorgeschlagen. }\end{array}$ \\
\hline 24.06 .2016 & $\begin{array}{l}\text { Außenminister Witold Waszczykowski bewertet das Ergebnis des Referendums in Großbritannien am Vortag, } \\
\text { aus der Europäischen Union auszuscheiden, als Warnsignal, das die EU-Mitglieder mobilisieren sollte. Not- } \\
\text { wendig sei die Reform der EU mit dem Ziel des Bürokratieabbaus und einer größeren demokratischen Legiti- } \\
\text { mierung der Entscheidungen auf EU-Ebene. Nun müsse weiteren Spaltungen in der EU vorgebeugt werden. }\end{array}$ \\
\hline
\end{tabular}




\begin{tabular}{|c|c|}
\hline 25.06 .2016 & $\begin{array}{l}\text { In einem Telefonat tauschen sich Außenminister Witold Waszczykowski und sein britischer Amtskollege Philip } \\
\text { Hammond über das Votum der Briten im Referendum am Vortag aus, aus der EU auszutreten. Hammond } \\
\text { bekräftigt, dass das Ergebnis des Referendums keinen Einfluss auf die Situation der polnischen Immigranten } \\
\text { in Großbritannien haben wird. In der Kampagne zur Volksabstimmung waren Ausländerfeindlichkeit und die } \\
\text { Ablehnung der Arbeitnehmerfreizügigkeit in der EU zutage getreten. }\end{array}$ \\
\hline 27.06.2016 & $\begin{array}{l}\text { In Prag treffen sich die Außenminister der Visegrád-Gruppe (Polen, Tschechien, Slowakei und Ungarn), Deutsch- } \\
\text { lands und Frankreichs. Thematisiert werden das Ergebnis und die Folgen des Referendums in Großbritannien in } \\
\text { der vergangenen Woche, in dem sich die Briten mehrheitlich für einen Austritt aus der EU ausgesprochen haben. }\end{array}$ \\
\hline 27.06 .2016 & $\begin{array}{l}\text { Auf Einladung von Außenminister Witold Waszczykowski kommen in Warschau die Außenminister von } \\
\text { Rumänien, Bulgarien, Griechenland und Ungarn sowie Staatssekretäre und hohe Beamte der Außenminis- } \\
\text { terien Spaniens, Sloweniens, der Slowakei und Österreichs, des Weiteren der Minister für europäische Ange- } \\
\text { legenheiten Großbritanniens zusammen. Es handele sich dabei nicht um die Schaffung eines festen Formats, } \\
\text { vielmehr seien in einem offenen Gedankenaustausch Ansichten und Bewertungen der Entscheidung Großbri- } \\
\text { tanniens für den EU-Austritt in einem Referendum in der vergangenen Woche ausgetauscht und Lösungen } \\
\text { vorgeschlagen worden, so Waszczykowski. Er ruft dazu auf, dass es solche Debatten, die niemanden ausschlie- } \\
\text { ßen, häufiger geben sollte, um die Einheit der EU zu wahren und gemeinsame Lösungen für Probleme zu fin- } \\
\text { den, die alle Mitgliedsländer beträfen. }\end{array}$ \\
\hline 28.06 .2016 & $\begin{array}{l}\text { In Posen (Poznań) gedenkt Präsident Andrzej Duda der Arbeitererhebung im Juni 1956, die militärisch nieder- } \\
\text { geschlagen worden war. Der Aufstand gilt als ein Bezugspunkt der oppositionellen Bewegung um Solidarność, } \\
\text { die in den 1980er Jahren zum Sturz des kommunistischen Systems beigetragen hat. Anwesend sind auch der } \\
\text { stellvertretende Ministerpräsident und Kulturminister Piotr Gliński, der Präsident Ungarns, János Áder, und } \\
\text { der ehemalige Solidarność-Führer und erste Präsident im demokratischen Polen, Lech Wałęsa. }\end{array}$ \\
\hline 29.06 .2016 & $\begin{array}{l}\text { Nach dem EU-Gipfel der Staats- und Regierungschefs infolge der Entscheidung Großbritanniens für den Aus- } \\
\text { tritt aus der EU unterstreicht Ministerpräsidentin Beata Szydło, dass es nun keinen Übergang zur Tagesord- } \\
\text { nung geben dürfe, sondern Fehlentwicklungen korrigiert und über die Zukunft der EU nachgedacht werden } \\
\text { müssen. Wichtig sei die Einheit, eine Union unterschiedlicher Geschwindigkeiten würde ihr Ende bedeuten. }\end{array}$ \\
\hline 30.06 .2016 & $\begin{array}{l}\text { Medienberichten zufolge einigt sich das Europäische Parlament darauf, die Debatte u. a. über die Situation des } \\
\text { Verfassungstribunals in Polen sowie die EU-Resolution zur Lage der Rechtsstaatlichkeit in Polen auf Septem- } \\
\text { ber zu verschieben. Grund dafür ist die Krise infolge der Entscheidung der Bürger Großbritanniens für den } \\
\text { EU-Austritt. }\end{array}$ \\
\hline 01.07.2016 & $\begin{array}{l}\text { Polen übernimmt regulär für ein Jahr den Vorsitz der Visegrád-Gruppe (Polen, Slowakei, Tschechien, Ungarn). } \\
\text { Angesichts der Krisen, mit denen die EU innerhalb und außerhalb der Union konfrontiert ist, könne die Vise- } \\
\text { grád-Gruppe die Befindlichkeiten der ostmitteleuropäischen EU-Mitgliedsländer wirksam repräsentieren und } \\
\text { die Debatte über die europäische Integration aktiv mitgestalten, heißt es im Programm für das kommende Jahr. } \\
\text { Zuletzt hatte die Tschechische Republik den Vorsitz inne. }\end{array}$ \\
\hline 02.07 .2016 & $\begin{array}{l}\text { In Warschau findet der Parteitag von Recht und Gerechtigkeit (Prawo i Sprawiedliwość - PiS) statt. Jarosław } \\
\text { Kaczyński, einziger Kandidat für die Wahl des Parteivorsitzenden, wird mit } 1.008 \text { Stimmen bei sieben Gegen- } \\
\text { stimmen und einer Enthaltung wiedergewählt. In seiner Parteitagsrede mahnt er insbesondere im Finanz-, } \\
\text { Gesundheits- und Außenressort Reformen an. PiS sei für eine Europäische Union als Union der Nationen. Für } \\
\text { die Erneuerung der EU sei die Überzeugung grundlegend, dass Europa von Nationen gebildet wird, die seit } \\
\text { Jahrhunderten als souveräne Staaten funktionieren. }\end{array}$ \\
\hline 03.07.2016 & $\begin{array}{l}\text { In einem Brief aus Anlass des 70. Jahrestags des Pogroms in Kielce unterstreicht Ministerpräsidentin Beata } \\
\text { Szydło, dass in Polen kein Platz für Rassismus sei und niemand wegen seines Glaubens verurteilt werden dürfe. } \\
\text { Bei dem Pogrom im Jahr } 1946 \text { wurden } 37 \text { Juden und drei Polen getötet. }\end{array}$ \\
\hline 04.07.2016 & $\begin{array}{l}\text { Wegen des NATO-Gipfels am 8. und 9. Juli in Warschau und des katholischen Weltjugendtags mit Papst Fran- } \\
\text { ziskus vom 26. bis } 31 \text {. Juli in Krakau führt Polen aus Sicherheitsgründen vorübergehend wieder Grenzkontrol- } \\
\text { len ein. Auch der »kleine Grenzverkehr" mit der russischen Exklave Kaliningrad und der Ukraine wird in die- } \\
\text { ser Zeit ausgesetzt. }\end{array}$ \\
\hline
\end{tabular}




\section{ÜBER DIE POLEN-ANALYSEN}

Die Polen-Analysen erscheinen zweimal monatlich als E-Mail-Dienst. Sie werden gemeinsam vom Deutschen PolenInstitut Darmstadt, der Bremer Forschungsstelle Osteuropa und der Deutschen Gesellschaft für Osteuropakunde herausgegeben.

Ein Archiv der Polen-Analysen finden Sie im Internet unter <www.laender-analysen.de/polen>

Kostenloses Abonnement unter <http://www.deutsches-polen-institut.de/Newsletter/subscribe.php >

Diese Analysen finden Sie online als Lizenzausgabe auf

$<$ bpb.de>

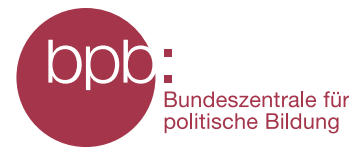

Deutsches Polen-Institut Darmstadt (<www.deutsches-polen-institut.de $>$ )

Das seit 1980 tätige Deutsche Polen-Institut Darmstadt (DPI) ist ein Forschungs-, Informations- und Veranstaltungszentrum für polnische Kultur, Geschichte, Politik, Gesellschaft und die deutsch-polnischen Beziehungen, die sich im Kontext der europäischen Integration entwickeln. Institutionelle Träger des DPI sind das Land Hessen, die Kultusminister der Länder, das Auswärtige Amt und die Wissenschaftsstadt Darmstadt. Einen wesentlichen Beitrag zur Verwirklichung der Institutsziele leisten private Stiftungen. Ziel der Vermittlertätigkeit des DPI ist es, »die zu interessieren, auf die es politisch, wirtschaftlich, gesellschaftlich und kulturell im deutsch-polnischen Verhältnis ankommt (Leitlinien 1997). Es geht um die Entscheider und Multiplikatoren in Politik, Kultur, Bildung, Verwaltung, Medien und Wirtschaft. Das DPI versteht sich in Kooperation mit den Orten wissenschaftlicher Polen-Kompetenz an deutschen Hochschulen und Forschungsinstituten als verbindendes und vernetzendes Zentrum. Mit der über 68.000 Bände zählenden multidisziplinären Fachbibliothek für Polen, die eine einzigartige Sammlung polnischer Belletristik in der Originalsprache und in deutscher Übersetzung umfasst, ist das DPI ein geschätzter Ort der Recherche und des wissenschaftlichen Arbeitens.

Forschungsstelle Osteuropa an der Universität Bremen (<www.forschungsstelle.uni-bremen.de>)

1982 gegründet, widmet sich die Forschungsstelle Osteuropa an der Universität Bremen der interdisziplinären Analyse der Länder Ost- und Ostmitteleuropas in Zeitgeschichte und Gegenwart. Der Forschungsschwerpunkt liegt dabei auf der Rolle von »Dissens und Konsens«, von Opposition und Zivilgesellschaft in ihrem historischen, politischen, gesellschaftlichen und kulturellen Kontext. Die Forschungsstelle besitzt in ihrem Archiv eine einzigartige Sammlung alternativer Kulturgüter und unabhängiger Texte aus den ehemaligen sozialistischen Ländern. Darunter befindet sich auch eine umfangreiche Sammlung des "Zweiten Umlaufs«, die das Schrifttum und Dokumente unabhängiger Initiativen und gesellschaftlicher Gruppen in Polen aus der Zeit von 1976 bis zum Umbruch umfasst. Hinzu kommt eine umfangreiche Bibliothek mit wissenschaftlicher Literatur. Mit Archiv, Bibliothek und zwei wissenschaftlichen Abteilungen ist die Forschungsstelle auch eine Anlaufstelle sowohl für Gastwissenschaftler als auch für die interessierte Öffentlichkeit.

Eine der Hauptaufgaben der Forschungsstelle ist die Information der interessierten Öffentlichkeit. Dazu gehören unter anderem regelmäßige E-Mail-Informationsdienste für Politik, Wirtschaft, Zivilgesellschaft und Medien.

Das Institut für Öffentliche Angelegenheiten (Instytut Spraw Publicznych - ISP) in Warschau ist einer der führenden Think Tanks in Polen und seit 1995 als unabhängiges Forschungszentrum zu grundlegenden Fragen des öffentlichen Lebens tätig. Das ISP kooperiert eng mit zahlreichen Experten und Forschern wissenschaftlicher Einrichtungen im In- und Ausland. <www.isp.org.pl>

Herausgegeben mit finanzieller Unterstützung der Stiftung für deutsch-polnische Zusammenarbeit
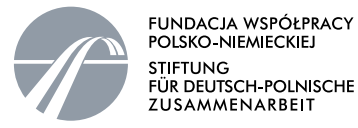

Die Meinungen, die in den Polen-Analysen geäußert werden, geben ausschließlich die Auffassung der Autoren wieder. Abdruck und sonstige publizistische Nutzung sind nach Rücksprache mit der Redaktion gestattet. Redaktion: Prof. Dr. Dieter Bingen (verantwortlich) (Darmstadt), Silke Plate M.A. (Bremen) Technische Gestaltung: Matthias Neumann

Polen-Analysen-Layout: Cengiz Kibaroglu, Matthias Neumann

Alle Ausgaben der Polen-Analysen sind mit Themen- und Autorenindex archiviert unter www.laender-analysen.de

Die Polen-Analysen werden im Rahmen der Datenbank World Affairs Online (WAO) ausgewertet und sind im Portal IREON www.ireon-portal.de recherchierbar.

ISSN 1863-9712 @ 2016 by Deutsches Polen-Institut Darmstadt und Forschungsstelle Osteuropa, Bremen

Kontakt: Dr. Andrzej Kaluza, Presse- und Öffentlichkeitsarbeit, Deutsches Polen-Institut, Residenzschloss, Marktplatz 15,

D-64283 Darmstadt, Tel.: +49/6151/4202-20, Fax: +49/6151/4202-10, E-Mail: polen-analysen@dpi-da.de, Internet: www.laender-analysen.de/polen 